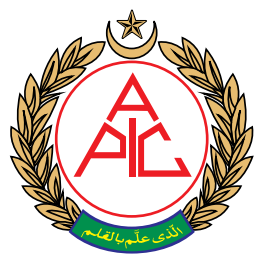

\title{
Prevalence of different pain patterns in patients with COVID-19: A systematic review and meta-analysis
}

\author{
Ali Gholami ${ }^{1,2}$, Mohammad Karimian ${ }^{3}$, Behzad Badakhsh ${ }^{4}$, \\ Feizollah Mansouri ${ }^{5}$, Mohamadreza Kafashian ${ }^{6}$, \\ Ali Khorshidi ${ }^{7}$, Behrouz Soltany ${ }^{8,2}$, Somayeh Mahdikhani ${ }^{9}$, \\ Milad Borji ${ }^{10,11}$, Asma Tarjoman ${ }^{10,11}$, Lida Nouri ${ }^{11}$
}

1-Assistant Professor of Anesthesiology, Department of Anesthesiology; 2-Student Research Committee, 5Professor of Infectious Disease, Infectious Diseases Department; 8-MSc Nursing, Department of Nursing, Faculty of Nursing and Midwifery, Kermanshah University of Medical Science, Kermanshah, IR Iran

3-Assistant Professor of Vascular Surgery; 4-Assistant Professor of Internal Medicine;

6-Associate Professor of Physiology, Department of Physiology; 7-Associate Professor of Epidemiology, School of Medicine, Psychosocial Injuries Research Center; 10-Student Research Committee; 11-Zoonotic Disease Research Center; 11 Assistant Professor of Anesthesiology, Department of Anesthesiology, Ilam University of Medical Sciences, Ilam, IR Iran

9-PhD Nursing Student, Università degli Studi "La Sapienza" di Roma, Public Health and Infectious Diseases, Undergraduate, (Italy)

Correspondence: $\mathrm{Dr}$ Lida Nouri, Assistant Professor of Anesthesiology, Department of Anesthesiology, Ilam University of Medical Sciences, Ilam, IR Iran. Tell: +98-9183404704,

E-mail:

lidanouri2016@gmail.com

Received: 15 March 2020,

Reviewed: 5 April 2020,

Accepted: 6 April 2020

\section{ABSTRACT}

Background and Objectives: Pain assessment is very important in these patients, but no comprehensive systematic reviews / meta-analyses (SRs/MAs) have been performed so far. For this reason, this study was performed to determine the prevalence of pain in patients with Covid-19 in the world by SR/MA method.

Methodology: The researchers collected English language articles in which COVID-19 was confirmed and all SRs/MAs and case reports articles were excluded. Search was carried on at SCOPUS ${ }^{\circledR}$, PubMed $^{\circledR} /$ MEDLINE $^{\circledR}$, Web of Science ${ }^{\circledR}$, Science Direct ${ }^{\circledR}$ and Google Scholar's search engine. To extract the data the checklist contained general information about articles, e.g. authors' names, year of publication, number of patients, country, journal's name, and specific information, e.g. prevalence and percentage of 'sore throat', 'abdominal pain', 'chest pain', 'headache' and 'myalgia'.

Results: According to the findings, 326 articles were extracted in the initial search, 218 articles of these were classified as duplicate articles because of the frequency in their authors, magazines and sample size, and were excluded. Also, by reviewing the title, abstract and complete files of articles, 73 articles were excluded as being non-relevant. Out of 35 remaining articles 2 were SRs/MAs in the field of COVID-19 by Iranian authors, and were also excluded. In the remaining 33 articles included in this SR/MA study, the sample size was 3781 patients.

Regarding the prevalence of pain in patients, prevalence rate of abdominal pain was $0.02 \%(95 \% \mathrm{Cl}: 0.01,0.04)$, headache $10 \% 95 \% \mathrm{Cl}: 0.10(0.08,0.12)$ and myalgia was $18 \% 95 \% \mathrm{Cl}$ : $0.18(0.14,0.23)$, chest pain was $4 \%(95 \% \mathrm{Cl}: 0.04$ $(0.01,0.06)$, Sore throat was $12 \%(95 \% \mathrm{Cl}: 0.12(0.08,0.15)$.

Conclusion: The results of this study can serve as important criteria to be considered for screening as well as identifying suspected cases of COVID-19. These can also be helpful in formulating the guidelines for the periodic physical evaluation and for clinical management of COVID-19 patients.

Key words: COVID-19; Pain, Coronavirus; Systematic review; Meta-analysis

Citation: Badakhsh B, Mansouri F, Gholami A, Karimian M, Kafashian M, Khorshidi A, Soltany B, Mahdikhani S, Borji M, Tarjoman A, Nouri L. Prevalence of different pain patterns in patients with COVID-19: A systematic review and meta-analysis. Anaesth. pain intensive care. 2020;24(2):141-150

DOI: https://doi.org/10.35975/apic.v24i2.1252 


\section{INTRODUCTION}

A significant population of the world has been affected by infectious diseases which have led to a decline in the quality of life and caused the death of these patients. ${ }^{1,2}$ These days COVID-19 is one of the most dangerous infectious diseases affecting the whole world. ${ }^{3-6}$ According to various studies that have been conducted in this field, symptoms of this disease may include fever, cough, nausea and/or vomiting, loss of sense of smell and taste, runny nose, fatigue and pain. The patients might also experience various types of pain, including sore throat, abdominal pain, chest pain, headache and myalgia., ${ }^{5,-9}$

Pain is one of the variables affecting health status that brings the patient to a hospital and helps the physician to early diagnosis of the disease. ${ }^{10-12}$ Considering, that the disease is a newfound illness, the presence of pain may not be taken seriously or might be mistaken to be originating due to other illnesses. Therefore, identifying clinical symptoms in this disease is very important and needs to be investigated and documented. ${ }^{13-15}$

\section{OBJECTIVES}

Pain assessment is very important in these patients, but no comprehensive SR/MA studies have been performed so far. For this reason, this study was performed to determine the prevalence of pain in patients with COVID-19 in the world by SR/MA method.

\section{METHODOLOGY}

\section{Protocol}

Researchers conducted a SR/MA type of study to determine the prevalence of pain in patients with COVID-19 according to the SR/MA statement (PRISMA) check list.16

\section{Inclusion and exclusion criteria}

The researchers collected English language articles in which COVID-19 disease was confirmed and all SRs/MAs and case reports were excluded.

\section{Information sources and search strategy}

Search was carried on at SCOPUS $®$, PubMed® / MEDLINE $®$, Web of Science ${ }^{\circ}$, Science Direct ${ }^{\circledR}$ and Google Scholar's search engines. Key words used for search included COVID-19, Novel coronavirus, SARS-CoV-2, Wuhan coronavirus, Novel coronavirus 2019, Wuhan pneumonia, $2019 \mathrm{nCoV}$ and pain or pain related terms including Sore throat, Abdominal pain, Chest pain, Headache and Myalgia.

\section{Study selection}

In the early searches, articles were reviewed for title and abstract by MB and AT, then full texts and references of articles that met the inclusion criteria of these article were studied and according to the existing checklist, required information was extracted. Duplicate articles or those containing incomplete information were excluded. After the search results finalized, articles extracted were reviewed and approved by a third researcher of the AG Study Team.

\section{Data collection process and data items}

To extract the data a tabulated form was used containing general information of articles (author's name, year of publication, number of patients, country, journal's name) and specific information (prevalence and percentage of Sore throat, Abdominal pain, Chest pain, Headache, Myalgia) (Table 1).

\section{Data analysis:}

Data were analyzed using Stata software.

\section{RESULTS}

According to the findings, 326 articles were extracted in the initial search, 218 of these were classified as duplicated articles, because of the multiple appearance of their author names, magazines and sample size. Also, by reviewing the title, abstract and complete file of 73 articles were excluded being non-relevant. The left over 35 articles were again analyzed and 2 Iranian SRs/MAs in the field of COVID-19, were also excluded (Figure 1). 
In the 33 articles included in this SR/MA study, the sample size was 3781 patients (Table 1). Regarding the prevalence of pain in patients, the prevalence of abdominal pain was $0.02 \%$ (95\% CI: $0.01,0.04)$, the prevalence of headache was $10 \%$ (95\% CI: $0.10(0.08,0.12)$, myalgia $18 \% \quad(95 \%$ CI : $0.18(0.14,0.23)$, chest pain was $4 \% \quad(95 \%$ CI : 0.04 $(0.01,0.06)$ and sore throat was $12 \%$ (95\% CI : $0.12(0.08,0.15)$ (Figures 2$6)$.

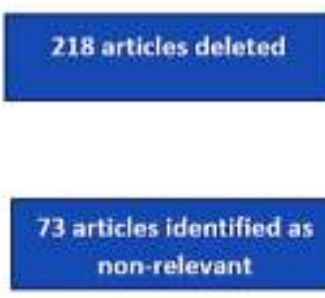

2 Iranean review articles excluded
Initially 326 articles

Identified and reviewed

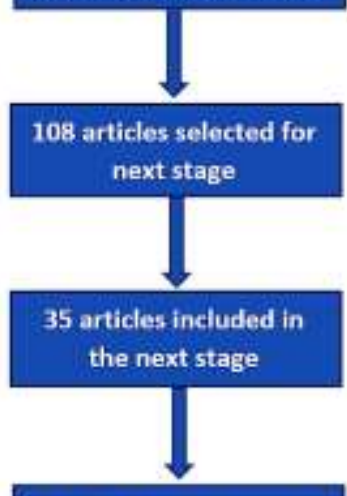

Finally 33 articles were analyzed

Figure 1: Flowchart for SR/MA

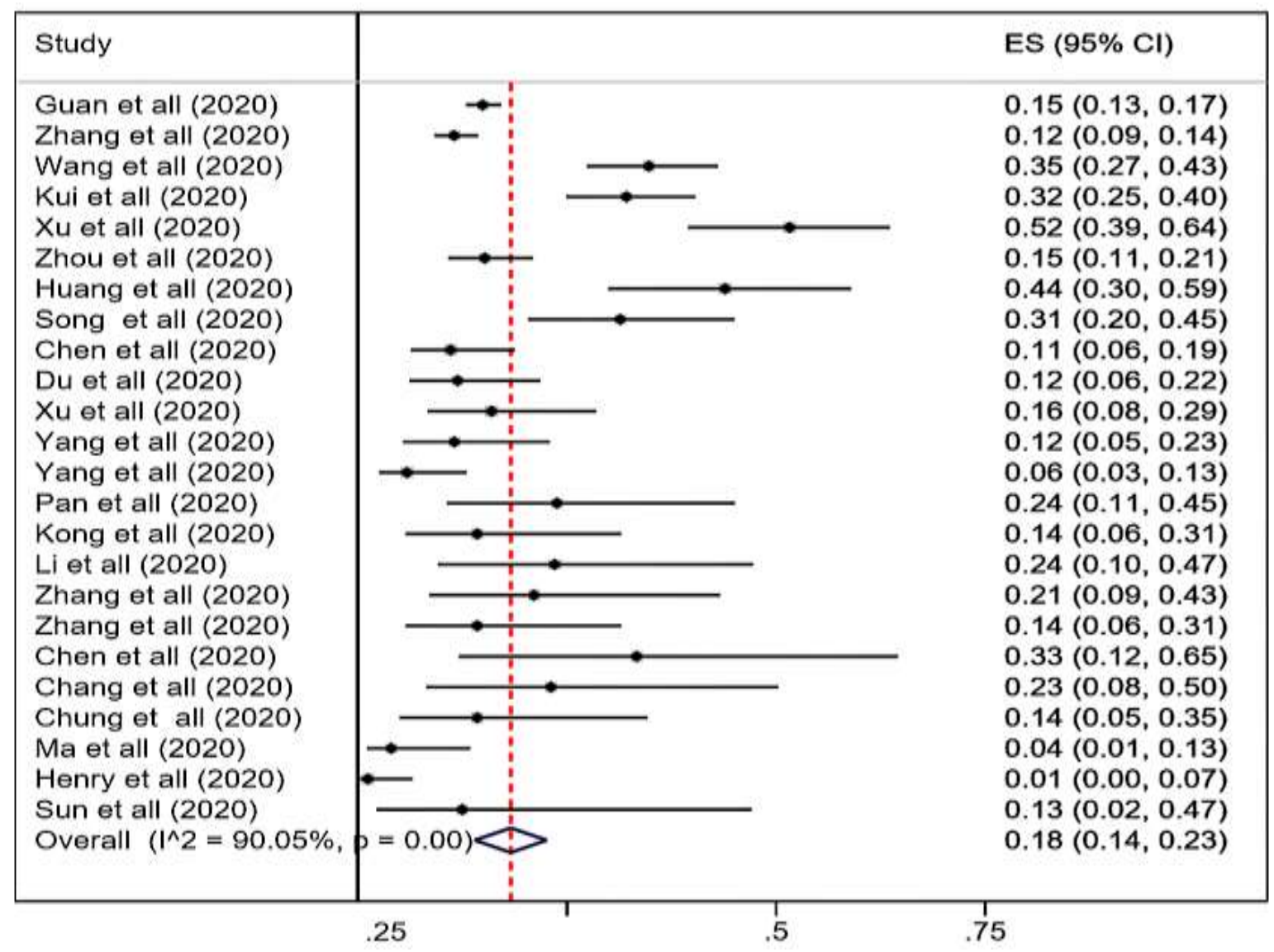

Figure 2: Prevalence of myalgia in studies entered into the SR/MA 


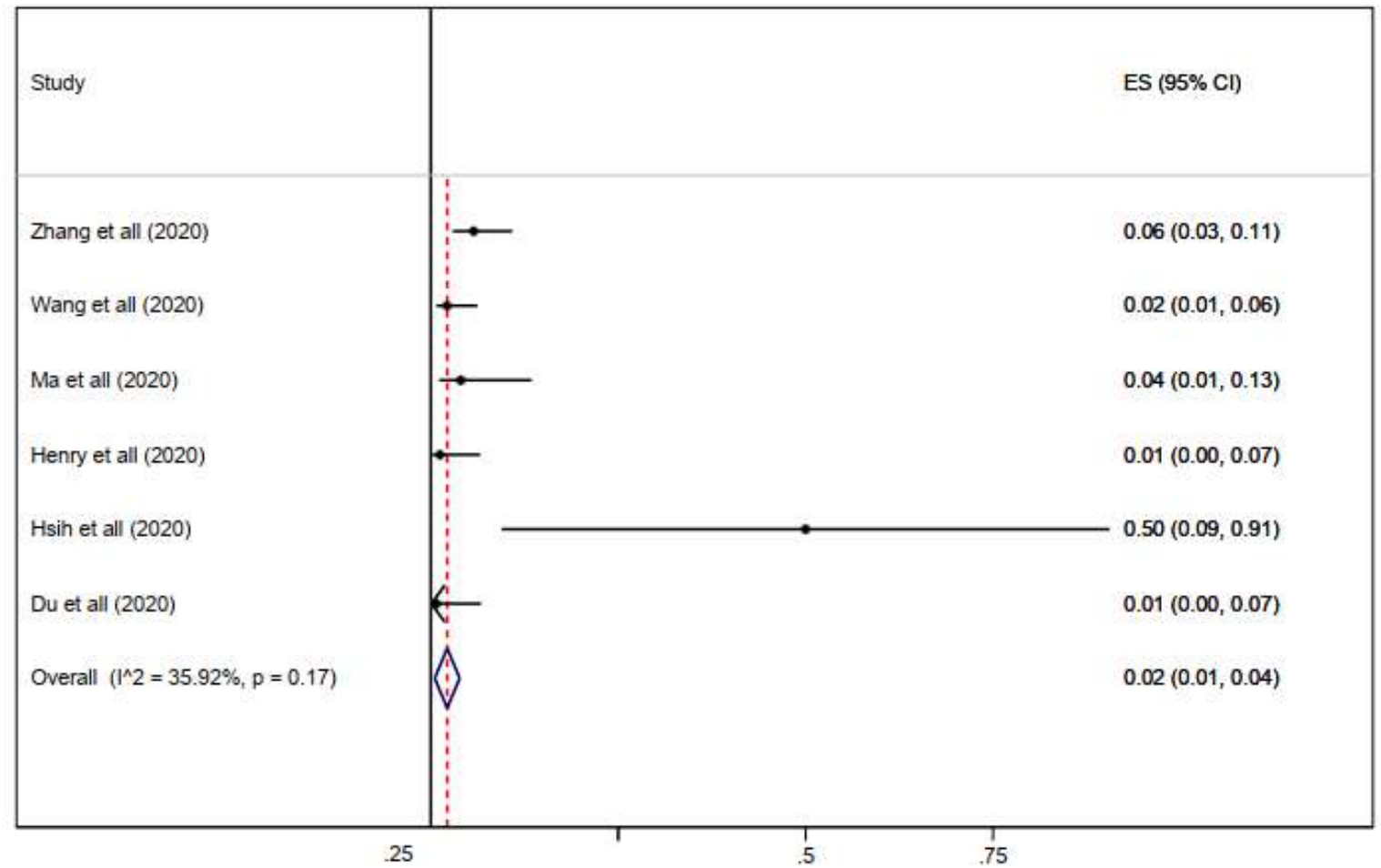

Figure 3: Prevalence of abdominal pain in studies entered into the SR/MA

\begin{tabular}{|c|c|c|}
\hline Study & & $\mathrm{ES}(95 \% \mathrm{Cl})$ \\
\hline Song et all (2020) & $\longrightarrow$ & $0.14(0.07,0.26)$ \\
\hline Yang et all (2020) & & $0.06(0.03,0.13)$ \\
\hline Chen et all (2020) & & $0.02(0.01,0.07)$ \\
\hline Pan et all $(2020)$ & & $0.10(0.03,0.29)$ \\
\hline Zhang et all (2020) & & $0.11(0.03,0.31)$ \\
\hline Zhang et all (2020) & & $0.07(0.02,0.23)$ \\
\hline $\mathrm{Ng} \mathrm{M}$ et all (.) & & $0.05(0.01,0.23)$ \\
\hline Yang et all (.) & & $0.02(0.00,0.10)$ \\
\hline Chen et all (.) & & $0.05(0.01,0.36)$ \\
\hline Ma et all (.) & & $0.01(0.00,0.09)$ \\
\hline Overall $\left(1^{\wedge} 2=26.22 \%, p=0.20\right)$ & & $0.04(0.01,0.06)$ \\
\hline
\end{tabular}

Figure 4: Prevalence of chest pain in studies entered into the SR/MA 


\begin{tabular}{|c|c|c|}
\hline Study & & $\mathrm{ES}(95 \% \mathrm{Cl})$ \\
\hline Guan et all (2020) & + & $0.14(0.12,0.16)$ \\
\hline Zhang et all (2020) & & $0.11(0.09,0.14)$ \\
\hline Chen et all (2020) & $\rightarrow$ & $0.11(0.08,0.16)$ \\
\hline Xu et all $(2020)$ & & $0.34(0.23,0.46)$ \\
\hline Tian et all (2020) & $\rightarrow$ & $0.06(0.04,0.10)$ \\
\hline Kui et all (2020) & 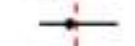 & $0.09(0.06,0.16)$ \\
\hline Yang et all (2020) & & $0.15(0.09,0.24)$ \\
\hline Du et all (2020) & & $0.18(0.11,0.29)$ \\
\hline Wang et all (2020) & & $0.07(0.03,0.12)$ \\
\hline Chen et all (2020) & $\rightarrow-i$ & $0.08(0.04,0.15)$ \\
\hline Song et all (2020) & & $0.16(0.08,0.28)$ \\
\hline Shi et all $(2020)$ & - & $0.06(0.03,0.14)$ \\
\hline Xu et all (2020) & & $0.10(0.04,0.21)$ \\
\hline Huang et all (2020) & $\rightarrow$ & $0.07(0.03,0.19)$ \\
\hline Chang et all (2020) & & $0.23(0.08,0.50)$ \\
\hline Kong et all (2020) & & $0.11(0.04,0.27)$ \\
\hline Chung et all (2020) & & $0.14(0.05,0.35)$ \\
\hline Yang et all (2020) & $\rightarrow$ & $0.06(0.02,0.16)$ \\
\hline Qui et all (2020) & & $0.08(0.03,0.22)$ \\
\hline Chen et all (2020) & $\rightarrow \quad 1$ & $0.03(0.01,0.16)$ \\
\hline Sun et all (2020) & 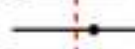 & $0.13(0.02,0.47)$ \\
\hline Overall $\left(1^{\wedge} 2=62.77 \%\right.$. & $=0,(0)$ & $0.10(0.08,0.12)$ \\
\hline
\end{tabular}

Figure 5: Prevalence of headache in studies entered into the SR/MA

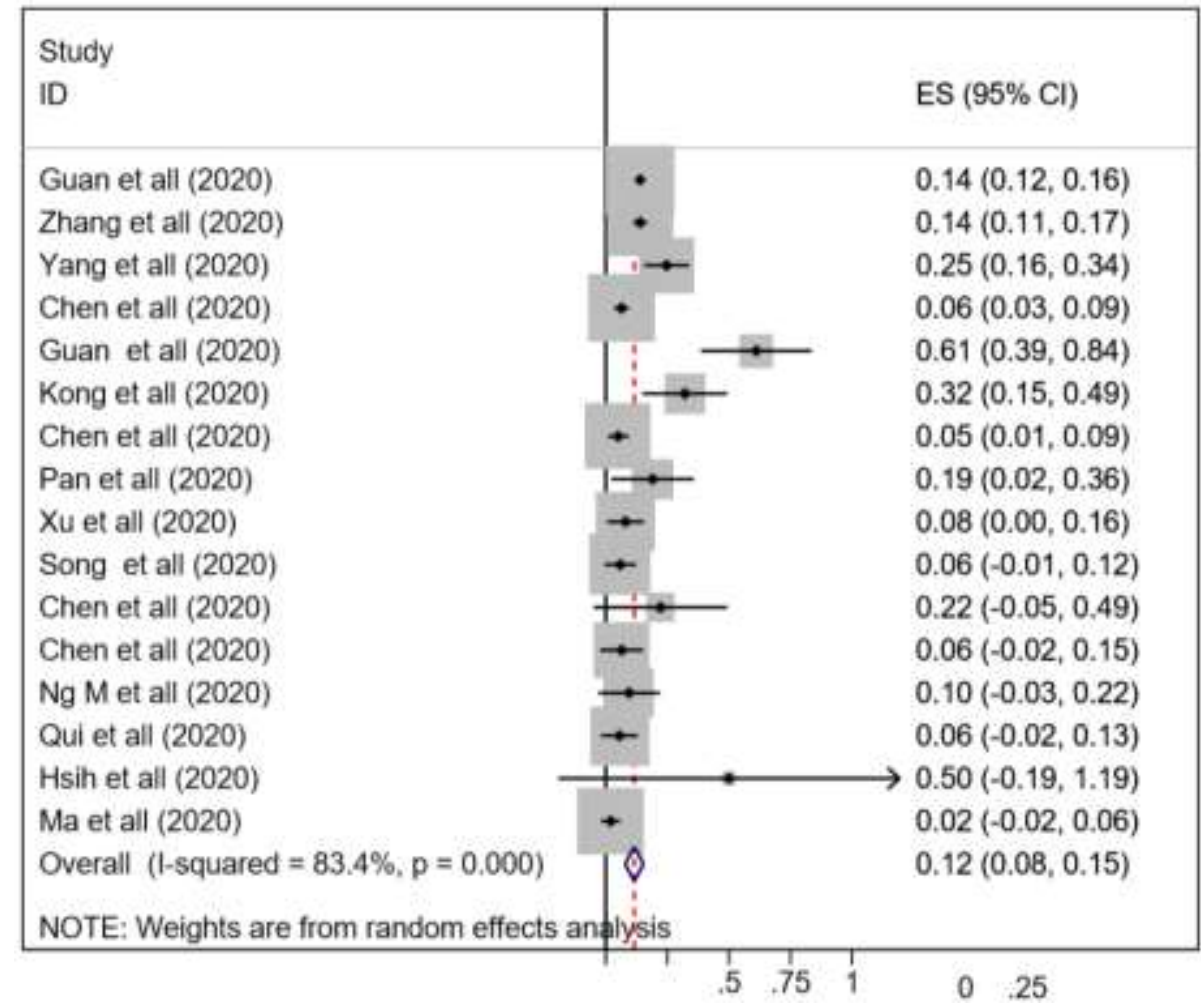

Figure 6: Prevalence of sore throat in studies entered into the SR/MA 
Table 1: Specifications of studies entered into the SR/MA

\begin{tabular}{|c|c|c|c|c|c|c|c|c|c|c|c|}
\hline Author & Age & Country & $\mathbf{N}$ & $\begin{array}{l}\text { Male } \\
\text { N (\%) }\end{array}$ & $\begin{array}{l}\text { Female } \\
\mathbf{N}(\%)\end{array}$ & Journal & $\begin{array}{l}\text { Myalgia } \\
\text { N (\%) }\end{array}$ & $\begin{array}{c}\text { Headach } \\
\text { e } \\
\text { N (\%) }\end{array}$ & $\begin{array}{c}\text { Chest } \\
\text { pain } \\
\mathrm{N}(\%)\end{array}$ & $\begin{array}{l}\text { Abdomi } \\
\text { nal pain }\end{array}$ & $\begin{array}{l}\text { Sore } \\
\text { throat }\end{array}$ \\
\hline 1 Huang et al. $(2020)^{17}$ & $49(41-58)$ & China & 41 & $30(73)$ & $11(27)$ & Lancet & $18(44)$ & $3(8)$ & & - & - \\
\hline 2 Chen et al. $(2020)^{18}$ & $55 \cdot 5(13 \cdot 1)$ & China & 99 & $67(68 \%)$ & $32(32 \%)$ & Lancet & $11(11)$ & $8(8)$ & $2(2)$ & - & $5(5)$ \\
\hline 3 Chen et al. $(2020)^{19}$ & 29.88 & China & 9 & - & - & Lancet & $3(33)$ & - & $0(0)$ & - & $2(22)$ \\
\hline 4 Wang et al. $(2020)^{20}$ & 56 & China & 138 & $63(45.7)$ & $75(54.3)$ & JAMA & $48(34.8)$ & $9(6.5)$ & -- & $3(2.2)$ & - \\
\hline 5 Guan et al. $(2020)^{21}$ & $47(31-73)$ & China & 18 & $9(50)$ & $9(50)$ & JAMA & - & - & - & - & $11(61)$ \\
\hline 6 Zhang et al. $(2020)^{22}$ & $57(25-87)$ & China & 140 & $71(50.7)$ & $69(49.3)$ & Al. ergy & - & - & - & $8(5.8)$ & - \\
\hline 7 Guan et al. $(2020)^{23}$ & $47(35-58)$ & China & 1099 & $640(58.1)$ & $459(41.9)$ & N Engl J Med & $\begin{array}{l}164 \\
(14.9)\end{array}$ & $\begin{array}{l}150 \\
(13.6)\end{array}$ & - & - & $\begin{array}{l}153 \\
(13.9)\end{array}$ \\
\hline 8 Kui et al. $(2020)^{24}$ & $57(20-83)$ & China & 137 & $61(44.5)$ & $76(55.5)$ & Chin Med J & $44(32.1)$ & $13(9.5)$ & - & - & - \\
\hline 9 Xu et al. $(2020)^{25}$ & $41(32-52)$ & China & 62 & $35(56)$ & $27(44)$ & BMJ & $32(52)$ & $21(34)$ & - & - & - \\
\hline 10 Chang et al. $(2020)^{26}$ & $34(34-48)$ & China & 13 & - & - & & $3(23.1)$ & $3(23.1)$ & - & - & - \\
\hline 11 Chen et al. $(2020)^{27}$ & $7.33 \pm 4.35$ & China & 31 & $13(41.9)$ & $18(58.1)$ & $\begin{array}{l}\text { Lancet Child } \\
\text { Adolesc Health }\end{array}$ & - & $1(3.2 \%)$ & - & - & $2(6.5)$ \\
\hline 12 Henry et al. $(2020)^{28}$ & $0-19$ & China & 82 & $43(52.4)$ & 27 (32.9) & - & $1(4)$ & - & - & $1(4)$ & - \\
\hline 13 Kong et al. $(2020)^{29}$ & $20-79$ & $\begin{array}{l}\text { South } \\
\text { Korea }\end{array}$ & 28 & $15(53.6)$ & $13(46.4)$ & $\begin{array}{l}\text { Osong Public } \\
\text { Health Res } \\
\text { Perspect }\end{array}$ & $4(14.3)$ & $3(10.7)$ & - & - & $9(32.1)$ \\
\hline 14 Yang et al. $(2020)^{30}$ & $\begin{array}{l}45.11 \pm \\
13.35\end{array}$ & China & 85 & 81 & 68 & J Infect & $5(3.36)$ & $13(8.72)$ & $5(3.36)$ & - & $\begin{array}{l}21 \\
(14.09)\end{array}$ \\
\hline 15 Li et al. $(2020)^{31}$ & $45.1 \pm 12.8$ & & 17 & $9(52.9)$ & $8(47.1)$ & & $4(23.5)$ & - & - & - & - \\
\hline 16 Pan et al. $(2020)^{32}$ & $\begin{array}{l}40 \pm 9(25- \\
63)\end{array}$ & China & 21 & $6(29 \%)$ & $15(74 \%)$ & Radiology & $5(24)$ & - & $2(9.5)$ & - & $4(19)$ \\
\hline
\end{tabular}


original research

\begin{tabular}{|c|c|c|c|c|c|c|c|c|c|c|c|}
\hline $17 \mathrm{Ng} \mathrm{M}$ et al. $(2020)^{33}$ & $56(37-65)$ & $\begin{array}{l}\text { Hong } \\
\text { Kong }\end{array}$ & 21 & $13(62)$ & $8(38)$ & & - & - & $1(5 \%)$ & - & $2(10 \%)$ \\
\hline 18 Tian et al. $(2020)^{34}$ & $47.5(1-94)$ & & 262 & $127(48.5)$ & $135(51.5)$ & J Infect & - & $17(6.5)$ & - & - & - \\
\hline 19 Song et al. $(2020)^{35}$ & $49 \pm 16$ & & 51 & $25(49)$ & $26(51)$ & & $16(31)$ & $8(16)$ & $7(14)$ & - & $3(6)$ \\
\hline 20 Chung et al. $(2020)^{36}$ & $51 \pm 14$ & China & 21 & - & - & Radiology & $3(14)$ & $3(14)$ & - & - & - \\
\hline 21 Zhou et al. $(2020)^{37}$ & $\begin{array}{l}56 \cdot 0(46 \cdot 0- \\
67 \cdot 0)\end{array}$ & China & 191 & $72(38)$ & $119(62)$ & Lancet & $29(15)$ & - & - & - & - \\
\hline 22 Shi et al. $(2020)^{38}$ & $49.5(11)$ & China & 81 & $42(52)$ & $39(48)$ & $\begin{array}{l}\text { Lancet Infect } \\
\text { Dis }\end{array}$ & - & $5(6)$ & - & - & - \\
\hline 23 Hsih et al. $(2020)^{39}$ & $45.0(39-51)$ & China & 2 & $1(50)$ & $1(50)$ & $\begin{array}{l}\text { J Microbiol } \\
\text { Immunol Infect }\end{array}$ & - & - & - & $1(50)$ & $1(50)$ \\
\hline 24 Yang et al. $(2020)^{40}$ & $59 \cdot 7(13 \cdot 3)$ & China & 52 & $35(67)$ & $17(33)$ & $\begin{array}{l}\text { Lancet Respir } \\
\text { Med }\end{array}$ & $6(11.5)$ & $3(6)$ & $1(2)$ & - & - \\
\hline 26 Qui et al. (2019) & 8.3 & China & 36 & $23(64)$ & $13(36)$ & $\begin{array}{l}\text { Lancet Infect } \\
\text { Dis }\end{array}$ & - & $3(8)$ & - & - & $2(6)$ \\
\hline 27 Du et al. $(2020)^{43}$ & 34.10 & China & 67 & $32(47.8)$ & $35(52.2)$ & $\begin{array}{l}\text { Lancet Infect } \\
\text { Dis }\end{array}$ & $8(11.9)$ & $12(17.9)$ & - & $0(0)$ & - \\
\hline $28 \mathrm{Xu}$ et II $(2020)^{44}$ & $43.9 \pm 16.8$ & China & 50 & $29(58)$ & $21(42)$ & J Infect & $8(16)$ & $5(10)$ & - & - & $4(8)$ \\
\hline 29 Zhang et al. (2020) & & & 19 & & & & $4(14.3)$ & - & $2(7.1)$ & - & - \\
\hline 30 Chen et al. $(2020)^{46}$ & $51(36-64)$ & China & 249 & $126(50.6)$ & $123(49.4)$ & J Infect & - & $28(11.2)$ & - & - & $16(6.4)$ \\
\hline 31 Zhang et al. (2020) & $\begin{array}{l}46.65 \pm \\
13.82 \\
\end{array}$ & & 573 & $295(51.5)$ & $278(48.5)$ & & $66(11.5)$ & $65(11.3)$ & - & - & $80(14)$ \\
\hline 32 Sun et al. $(2020)^{48}$ & - & China & 8 & $6(75)$ & $2(25)$ & World J Pediatr & $1(12.5)$ & $1(12.5)$ & - & - & - \\
\hline 33 Zhang et al. $(2020)^{49}$ & $65.0(56-70)$ & China & 28 & $17(60.7)$ & $11(39.3)$ & Ann Oncol & 4 (14.3) & - & $2(7.1)$ & & \\
\hline
\end{tabular}




\section{DISCUSSION}

Patients with COVID-19, experience a wide range of symptoms including fever, chills, nausea, vomiting, diarrhea, coughing and runny nose.50,51 Pain is another symptom of these patients which in this study has been investigated specifically.

This SRs/MAs study investigated pain in 33 articles in which, for the first time in the world, the pain of patients with COVID-19 was evaluated. In the SRs/MAs study published by Nasiri et al, the prevalence of Myalgia in 17 articles was 34.7 (26.0-44.4), headache prevalence in 12 articles was 11.1 (7.7-15.7) and sore throat was $14.5(10.6-19.5) 52$. While in this study, the prevalence of Myalgia in 28 analyzed articles was 18\% (95\% CI : $0.18(0.14$, 0.23) 'Headache prevalence in 21 articles was equal $10 \%(95 \%$ CI : $0.10(0.08,0.12)$ and the prevalence of sore throat with analyze of 16 articles was $12 \%$ (95\% CI : $0.12(0.08,0.15)$. It seems that regard to more articles have entered SRs/MAs phase and larger sample size, this study has been able to provide more accurate information.

This study investigated the specificity of pain as an important variable in patients' health status which is the innovation of this study. One of the disadvantages of this study is the lack of evaluation of pain status based on the demographic characteristics of the patients. For this reason, it is suggested that by updating articles on clinical symptoms and according to types of pain in patients with COVID-19, in future studies analyze accomplish according to demographic characteristics of patients and underlying diseases.

\section{CONCLUSION}

The results of this study can serve as an important criterion in evaluation of patients' health status also consider for screening as well as identifying suspected cases of COVID-19.

Acknowledgment: Student Research Committee, Kermanshah University of Medical Sciences, Kermanshah, Iran (Grant Number: 3009965)

Conflict of interest: The authors have no conflicts of interest to disclose.

Author contributions

AG, LLN BB: Manuscript writing

MK, FM: Editing

MK, AK: Analysed

$B S, M B, A T$ : Literature search

SM : Translation

\section{REFERENCES}

1. Tenforde MW, Gertz AM, Lawrence DS, Wills NK, Guthrie BL, Farquhar $C$, et al. Mortality from HIVassociated meningitis in subSaharan Africa: a systematic review and meta-analysis. J Int AIDS Soc. 2020 Jan;23(1):e25416. [PubMed] DOI: $10.1002 / j i a 2.25416$

2. Carignan A, Denis M, Abou Chakra. Mortality associated with Blastomyces dermatitidis infection: A systematic review of the literature and meta-analysis. Med Mycol. 2020 Jan 1;58(1):1-10. [PubMed] DOI: $10.1093 / \mathrm{mmy} / \mathrm{myz} 048$

3. Guo H, Zhou Y, Liu X, Tan J. The impact of the COVID-19 epidemic on the utilization of emergency dental services. J Dent Sci. 2020
Mar 16. [PubMed] DOI: 10.1016/i.jds.2020.02.002

4. Lippi G, Plebani M, Henry B. Thrombocytopenia is associated with severe coronavirus disease 2019 (COVID-19) infections: A meta-analysis. Clin Chim Acta. 2020 Mar 13:506:145-8. [PubMed] DOI: 10.1016/i.cca.2020.03.022

5. Zhang J, Yan K, Ye H, Lin J, Zheng $J$, Cai T. SARS-CoV-2 turned positive in a discharged patient with COVID-19 arouses concern regarding the present standard for discharge. Int J Infect Dis. 2020 Mar 18. pii: S1201-9712(20)30126-0. [PubMed] DOI: 10.1016/j.jijid.2020.03.007

6. Nemati M, Ebrahimi B, Nemati F. Assessment of Iranian nurses' knowledge and anxiety toward COVID-19 during the current outbreak in Iran. Arch Clin Infect Dis. 2020;15(COVID-19):e102848. DOI: 10.5812/archcid.102848

7. Pang J, Wang MX, Ang IYH, Tan SHX, Lewis RF, Chen JI-P, et al. Potential rapid diagnostics, vaccine and therapeutics for 2019 novel Coronavirus (2019-ncoV): a systematic review. J Clin Med. 2020 Feb 26;9(3):pii: E623. [PubMed] DOI: $10.3390 / j \mathrm{~cm} 9030623$

8. Rothan HA, Byrareddy. The epidemiology and pathogenesis of coronavirus disease (COVID-19) outbreak. J Autoimmun. 2020 May;109:102433. [PubMed] DOI: 10.1016/j.jaut.2020.102433 
9. Cortegiani A, Ingoglia G, Ippolito M, Giarratano A, Einav S. A systematic review on the efficacy and safety of chloroquine for the treatment of COVID-19. J Crit Care. 2020 Mar 10. pii: S0883-9441(20)30390-7. [PubMed] 10.1016/j.jcrc.2020.03.005

10. Vasigh $A$, Jaafarpour $M$, Khajavikhan J, Khani A. The effect of gabapentin plus celecoxib on pain and associated complications after laminectomy. J Clin Diagn Res. $2016 \quad$ Mar; 10(3):UC04-8. [PubMed] DOI: 10.7860/JCDR/2016/17923.7346

11. Bastami M, Azadi A, Mayel M. The use of ice pack for pain associated with arterial punctures. J Clin Diagn Res. 2015 Aug;9(8):JC07-9. [PubMed] DOI: 10.7860/JCDR/2015/12657.6336

12. Jin P, Tseng LA, Zhang Y. Chronic low back pain: improving approach to diagnosis and treatment. In: Spine Pain Care. Springer; 2020. p.513-30.

13. Su VYF, Yang Y, Yang K, Chou K, Su W, Chen $Y$, et al. The Risk of death in 2019 novel coronavirus disease (COVID-19) in Hubei province. $2020.2 \mathrm{DOI}$ 10.2139/ssrn.3539655

14. Jiang F, Deng L, Zhang L, Cai $Y$, Cheung CW, Xia Z. Review of the clinical characteristics of coronavirus disease 2019 (COVID19). J Gen Intern Med. 2020 May;35(5):1545-9. [PubMed] DOI: 10.1007/s11606-020-05762-w

15. Nemati M, Ebrahimi B, Nemati $F$ : Assessment of Iranian nurses' knowledge and anxiety toward COVID-19 during the current outbreak in Iran. Arch Clin Infect Dis. 2020;15(COVID-19):e102848. DOI: 10.5812 /archcid. 102848

16. Moher D, Altman DG, Tetzlaff J, Liberati A. Preferred reporting items for systematic reviews and metaanalyses: the PRISMA statement. BMJ. 2009 Jul 21;339:b2535. [PubMed] DOI: 10.1136/bmj.b2535

17. Huang $C$, Wang $Y$, Li X, Ren L, Zhao J, Hu Y, et al. Clinical features of patients infected with 2019 novel coronavirus in Wuhan, China. Lancet. $\quad 2020 \quad$ Feb 15;395(10223):497-506. [PubMed]
DOI:

6736(20)30183-5

18. Chen N, Zhou M, Dong X, Qu J, Gong $F$, Han $Y$, et al. Epidemiological and clinical characteristics of 99 cases of 2019 novel coronavirus pneumonia in Wuhan, China: a descriptive study. Lancet. $\quad 2020 \quad$ Feb 15;395(10223):507-13. [PubMed] DOI: $\quad$ 10.1016/S01406736(20)30211-7

19. Chen H, Guo J, Wang C, Luo F, Yu $X$, Zhang $W$, et al. Clinical characteristics and intrauterine vertical transmission potential of COVID-19 infection in nine pregnant women: a retrospective review of medical records. Lancet. $2020 \mathrm{Mar}$ 7;395(10226):809-15. [PubMed] DOI: $\quad 10.1016 / 50140-$ 6736(20)30360-3

20. Wang D, Hu B, Hu C, Zhu F, Liu X, Zhang $J$, et al. Clinical characteristics of 138 hospitalized patients with 2019 novel coronavirus-infected pneumonia in Wuhan, China. JAMA. 2020 Feb 7;323(11):1061-9. [PubMed] DOI: 10.1001/jama.2020.1585

21. Young BE, Ong SWX, Kalimuddin S, Low JG, Tan SY, Loh J, et al. Epidemiologic features and clinical course of patients infected with SARS-CoV-2 in Singapore. JAMA. 2020 Mar 3;323(15):1488-94. [PubMed] DOI: 10.1001/jama.2020.3204

22. Zhang JJ, Dong X, Cao YY, Yuan $Y D$ Yang YB, Yan YQ, et al. Clinical characteristics of 140 patients infected by SARS-CoV-2 in Wuhan, China. Allergy. 2020 Feb 19;00:112. [PubMed] DOI: 10.1111/all.14238

23. Guan W, Ni Z, Hu Y, Liang W, Ou $\mathrm{C}$, $\mathrm{He} \mathrm{J}$, et al. Clinical characteristics of coronavirus disease 2019 in China. N Engl J Med. 2020;382:1708-20. DOI: 10.1056/NEJMoa2002032

24. Liu K, Fang YY, Deng Y, Liu W, Wang MF, Ma JP, et al. Clinical characteristics of novel coronavirus cases in tertiary hospitals in Hubei Province. Chin Med J (Engl). 2020 May 5;133(9):1025-31. [PubMed] DOI: 10.1097/CM9.0000000000000744
25. Xu XW, Wu XX, Jiang XG, Xu KJ, Ying LJ, Ma CL, et al. Clinical findings in a group of patients infected with the 2019 novel coronavirus (SARS-Cov-2) outside of Wuhan, China: retrospective case series. BMJ. 2020 Feb 19;368:m606. [PubMed] DOI: 10.1136/bmi.m606

26. Chang D, Lin M, Wei L, Xie L, Zhu G, Cruz CSD, et al. Epidemiologic and clinical characteristics of novel coronavirus infections involving 13 patients outside Wuhan, China. 2020.

27. Chen $C$, Cao M, Peng L, Guo X, Yang F, Wu W, Chen L, Yang Y, Liu $Y$, Wang FJC: Coronavirus Disease-19 Among Children Outside Wuhan, China. JAMA. 2020 Feb 7;323(11):1092-3. [PubMed] DOI: $10.1001 /$ jama.2020.1623

28. Henry BM, Oliveira MHS. Preliminary epidemiological analysis on children and adolescents with novel coronavirus disease 2019 outside Hubei Province, China: an observational study utilizing crowdsourced data. 2020. DOI: 10.1101/2020.03.01.20029884

29. Kong I, Park Y, Woo Y, Lee J, Cha $J$, Choi J, et al. Early epidemiological and clinical characteristics of 28 cases of coronavirus disease in South Korea. Osong Public Health Res Perspect. 2020 Feb;11(1):8-14. [PubMed] DOI: $10.24171 /$ j.phrp.2020.11.1.03

30. Yang W, Cao Q, Qin L, Wang X, Cheng Z, Pan A, et al. Clinical characteristics and imaging manifestations of the 2019 novel coronavirus disease (COVID-19): A multi-center study in Wenzhou city, Zhejiang, China. J Infect. 2020 Apr;80(4):388-93. [PubMed] DOI: 10.1016/j.jinf.2020.02.016

31. Li J, Li S, Cai Y, Liu Q, Li X, Zeng Z, et al. Epidemiological and clinical characteristics of 17 hospitalized patients with 2019 novel coronavirus infections outside Wuhan, China. 2020. DOI: 10.1101/2020.02.11.20022053

32. Pan F, Ye T, Sun $P$, Gui $S$, Liang $B$, $\mathrm{Li} L$, et al. Time course of lung changes on chest CT during recovery from 2019 novel coronavirus (COVID-19) pneumonia. Radiology. 
2020:200370.

DOI:

10.1148/radiol.2020200370

33. Ng MY, Lee EY, Yang J, Yang F, Li $\mathrm{X}$, Wang $\mathrm{H}$, et al. Imaging profile of the COVID-19 infection: radiologic findings and literature review. Radiol Cardiothorac Imaging. 2020;2(1). DOI: 10.1148/ryct.2020200034

34. Tian S, Hu N, Lou J, Chen K, Kang $X$, Xiang Z, et al. Characteristics of COVID-19 infection in Beijing. J Infec. 2020;80(4):401-6. DOI: 10.1016/j.jinf.2020.02.018

35. Song F, Shi N, Shan F, Zhang Z, Shen J, Lu H, et al. Emerging 2019 novel coronavirus (2019-nCoV) pneumonia. Radiology. 2020 Apr;295(1):210-7. [PubMed] DOI: 10.1148/radiol.2020200274

36. Chung $M$, Bernheim $A$, Mei $X$, Zhang $N$, Huang $M$, Zeng $X$, et al. CT imaging features of 2019 novel coronavirus (2019-nCoV). Radiology. 2020 Apr;295(1):202-7. [PubMed] DOI: 10.1148/radiol.2020200230

37. Zhou F, Yu T, Du R, Fan G, Liu Y, Liu Z, et al. Clinical course and risk factors for mortality of adult inpatients with COVID-19 in Wuhan, China: a retrospective cohort study. Lancet. $2020 \quad$ Mar 28;395(10229):1054-62. [PubMed] DOI: $\quad 10.1016 / S 0140-$ $\underline{6736(20) 30566-3}$

38. Shi $H$, Han $X$, Jiang N, Cao $Y$, Alwalid $O, G u$ J, et al. Radiological findings from 81 patients with COVID-19 pneumonia in Wuhan, China: a descriptive study. Lancet Infect Dis. 2020 Apr;20(4):425-34. [PubMed] DOI: 10.1016/S14733099(20)30086-4

39. Hsih WH, Cheng MY, Ho MW, Chou $\mathrm{CH}$, Lin PC, Chi CY, et al. Featuring COVID-19 cases via screening symptomatic patients with epidemiologic link during flu season in a medical center of central Taiwan. J Microbiol Immunol Infect.
2020 Mar 13. pii: S16841182(20)30068-2. [PubMed] DOI: 10.1016/i.jmii.2020.03.008

40. Yang X, Yu Y, Xu J, Shu H, Liu H, Wu $Y$, et al. Clinical course and outcomes of critically ill patients with SARS-CoV-2 pneumonia in Wuhan, China: a single-centered, retrospective, observational study. Lancet Respir Med. 2020 May;8(5):475-81. [PubMed] DOl: 10.1016/S2213-2600(20)30079-5

41. Ma H, Hu J, Tian J, Zhou X, Li H, Laws MT, et al. Visualizing the novel coronavirus (COVID-19) in children: what we learn from patients at Wuhan Children's Hospital. $2020 . \quad$ DOI: 10.2139/ssrn.3556676

42. Qiu H. Clinical and epidemiological features of 36 children with coronavirus disease 2019 (COVID19) in Zhejiang, China: an observational cohort study. Lancet Infect Dis. 2020. DOI: 10.1016/S1473-3099(20)30198-5

43. Du W, Yu J, Wang $H$, Zhang $X$, Zhang S, Li $Q$, et al. Clinical characteristics of COVID-19 in children compared with adults outside of Hubei Province in China. Infection. 2020 Apr 16. [PubMed] DOI: 10.1007/s15010-020-01427-2

44. Xu YH, Dong JH, An WM, Lv XY, Yin XP, Zhang JZ, et al. Clinical and computed tomographic imaging features of novel coronavirus pneumonia caused by SARS-CoV2. J Infect. 2020 Apr;80(4):394-400. [PubMed] DOI: 10.1016/j.jinf.2020.02.017

45. Zhang L, Zhu F, Xie L, Wang C, Wang J, Chen R, et al. Clinical characteristics of COVID-19infected cancer patients: A retrospective case study in three hospitals within Wuhan, China. Ann Oncol. 2020 Mar 26. pii: S09237534(20)36383-3. [PubMed] DOI: 10.1016/j.annonc.2020.03.296
46. Chen J, Qi T, Liu L, Ling Y, Qian Z, $\mathrm{Li} \mathrm{T}$, et al. Clinical progression of patients with COVID-19 in Shanghai, China. J Infect. 2020 May;80(5):e1-e6. [PubMed] DOI: 10.1016/j.jinf.2020.03.004

47. Zhang X, Cai H, Hu J, Lian J, Gu J, Zhang $S$, et al. Epidemiological, clinical characteristics of cases of SARS-CoV-2 infection with abnormal imaging findings. Int $\mathrm{J}$ Infect Dis. 2020 Mar 20;94:81-7. [PubMed] DOI: 10.1016/.ijid.2020.03.040

48. Sun D, Li H, Lu XX, Xiao H, Ren J, Zhang FR, et al. Clinical features of severe pediatric patients with coronavirus disease 2019 in Wuhan: a single center's observational study. World J Pediatr. 2020. [PubMed] DOI: 10.1007/s12519-020-00354-4

49. Zhang L, Zhu F, Xie L, Wang C, Wang $J$, Chen $R$, et al. Clinical characteristics of COVID-19infected cancer patients: a retrospective case study in three hospitals within Wuhan, China. Ann Oncol. 2020. [PubMed] DOI: 10.1016/j.annonc.2020.03.296

50. Gu J, Han B, Wang J. COVID-19: Gastrointestinal manifestations and potential fecal-oral transmission. Gastroenterology. 2020 May;158(6):1518-9. [PubMed] DOI: 10.1053/j.gastro.2020.02.054

51. Zu ZY, Jiang MD, Xu PP, Chen W, $\mathrm{Ni} Q Q$, Lu GM, et al. Coronavirus disease 2019 (COVID-19): A perspective from China. Radiology. 2020 Feb 21:200490. [PubMed] DOI: $10.1148 /$ radiol.2020200490

52. Nasiri MJ, Haddadi S, Tahvildari A, Farsi Y, Arbabi M, Hasanzadeh S, et al. COVID-19 clinical characteristics, and sex-specific risk of mortality: Systematic Review and Meta-analysis. $2020 . \quad$ DOI: $\underline{10.1101 / 2020.03 .24 .20042903}$ 